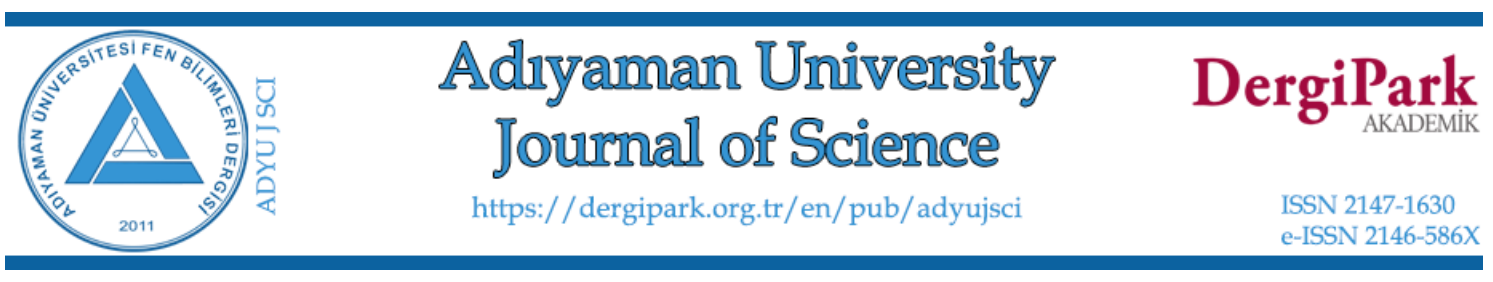

\title{
An Application of Subclasses of Harmonic Univalent Functions Involving
}

\section{Hypergeometric Function}

\author{
Waggas Galib ATSHAN ${ }^{1}$, Enaam Hadi ABD ${ }^{2,3}$, Sibel YALÇIN ${ }^{4, *}$ \\ ${ }^{I}$ Department of Mathematics, College of Science, University of Al-Qadisiyah, Diwaniya, Iraq \\ waggashnd@gmail.com,waggas.galib@qu.edu.iq,ORCID:0000-0002-7033-8993 \\ ${ }^{2}$ Department of Computer, College of Science, University of Kerbala, Kerbala, Iraq \\ ${ }^{3}$ Department of Mathematics, College of Science, University of Baghdad, Baghdad, Iraq \\ enaam_hadi2004@yahoo.com,ORCID:0000-0003-3580-8379 \\ ${ }^{4}$ Department of Mathematics, Faculty of Arts and Science, Uluda $\breve{g}$ University, Bursa, Turkey \\ syalcin@uludag.edu.tr,ORCID:0000-0002-0243-8263
}

\section{Abstract}

The main purpose of this paper is to establish connections between various subclasses of harmonic univalent functions by applying certain convolution operator involving hypergeometric functions. We investigate such connections with GoodmanSalagean-Type harmonic univalent functions in the open unit disc U.

Keywords: Univalent function; Uniformly convex; Linear operator; Hadamard product.

\section{Hipergeometrik Fonksiyonu İçeren Harmonik Tek Değerlikli Fonksiyonların}

\section{Altsınıflarının Bir Uygulaması}

\section{$\ddot{O} z$}

Bu makalenin amacı, hipergeometrik fonksiyonları içeren belirli konvolusyon operatörünü uygulayarak harmonik univalent fonksiyonların çeşitli altsınıfları arasında bağlantılar kurmaktır. 
Bu tür bağlantılar açık birim disk $U$ da Goodman-Salagean tipli harmonik univalent fonksiyonları ile araştırılmıştır.

Anahtar Kelimeler: Univalent fonksiyon; Düzgün konveks; Lineer operatör; Hadamard çarpımı.

\section{Introduction}

Let $A$ denote the class of analytic functions of the form:

$$
f(z)=z+\sum_{k=2}^{\infty} a_{k} z^{k},\left(a_{k} \geq 0, \mathrm{k} \in N\right),
$$

which is univalent in the open unit disc $\mathrm{U}=\{z \in C:|z|<1\}$. Hohlov [1] introduced the convolution operator $H(a, b ; c): A \rightarrow A$ defined by

$$
H(a, b ; c) f(z)=z F(a, b ; c ; z) * f(z),
$$

where $F(a, b ; c ; z)$ is a well-known Gaussian hypergeometric function and defined by

$$
F(a, b ; c ; z)=\sum_{k=0}^{\infty} \frac{(a)_{k}(b)_{k}}{(c)_{k}(1)_{k}} z^{k}
$$

where $a, b, c$ are complex numbers such that $c \neq 0,-1,-2, \ldots$.

A hypergeometric function $F(a, b ; c ; z)$ is analytic in $\mathrm{U}$ and plays an important role in Geometric Function Theory. See the studies by Branges [2], Ahuja [3], Carleson and Shaffer [4], Owa and Srivastava [5], Miller and Mocanu [6], Ruscheweyh and Singh [7], Srivastava and Manocha [8], and Swaminathan [9].

For a function $f \in A$ given by Eqn. (1) and $g \in A$ defined by

$$
g(z)=z+\sum_{k=2}^{\infty} b_{k} z^{k}
$$

we define the Hadamard product of $f$ and $g$ by

$$
(f * g)(z)=z+\sum_{k=2}^{\infty} a_{k} b_{k} z^{k} \quad, \quad z \in U
$$

Let $E$ be the family of all harmonic functions $f=h+\bar{g}$, where

$$
h(z)=z+\sum_{k=2}^{\infty} A_{k} z^{k} \quad, \quad g(z)=\sum_{k=1}^{\infty} B_{k} z^{k}, \quad\left|B_{1}\right|<1, z \in U
$$


are in the class $A$. For complex parameters $a_{1}, b_{1}, c_{1}, a_{2}, b_{2}, c_{2}\left(c_{1}, c_{2} \neq 0,-1,-2, \ldots\right)$, we define the functions $\Phi_{1}=z F\left(a_{1}, b_{1} ; c_{1} ; z\right)$ and $\Phi_{2}=z F\left(a_{2}, b_{2} ; c_{2} ; z\right)$.

Corresponding to these functions, we consider the following convolution operator

$$
\Omega \equiv \Omega\left(\begin{array}{lll}
a_{1}, & b_{1}, & c_{1} \\
a_{2}, & b_{2}, & c_{2}
\end{array}\right): E \rightarrow E
$$

defined by

$$
\Omega\left(\begin{array}{lll}
a_{1}, & b_{1}, & c_{1} \\
a_{2}, & b_{2}, & c_{2}
\end{array}\right) f=f *\left(\Phi_{1}+\overline{\Phi_{2}}\right)=h * \Phi_{1}+\overline{g * \Phi_{2}}
$$

for any function $f=h+\bar{g}$ in $E$. Letting

$$
\Omega\left(\begin{array}{lll}
a_{1}, & b_{1}, & c_{1} \\
a_{2}, & b_{2}, & c_{2}
\end{array}\right) F(z)=H(z)+\overline{G(z)}
$$

we have

$$
\begin{aligned}
& H(z)=z+\sum_{k=2}^{\infty} \frac{\left(a_{1}\right)_{k-1}\left(b_{1}\right)_{k-1}}{\left(c_{1}\right)_{k-1}(1)_{k-1}} A_{k} z^{k} \\
& G(z)=\sum_{k=1}^{\infty} \frac{\left(a_{2}\right)_{k-1}\left(b_{2}\right)_{k-1}}{\left(c_{2}\right)_{k-1}(1)_{k-1}} B_{k} z^{k} .
\end{aligned}
$$

We observe that

$$
\Omega\left(\begin{array}{lll}
a_{1}, & 1, & a_{1} \\
a_{2}, & 1, & a_{2}
\end{array}\right) f(z)=f(z)=f(z) *\left(\frac{z}{1-z}+\overline{\frac{z}{1-z}}\right)
$$

is the identity mapping.

This convolution operator $\Omega$ were defined and studied by the author in [10]. Denote by $S_{E}$ the subclass of $E$ that are univalent and sense-preserving in $\mathrm{U}$.

$$
\begin{aligned}
& \text { Note that } \frac{f-\overline{B_{1} f}}{1-\left|B_{1}\right|^{2}} \in S_{E} \text { whenever } f \in S_{E} \text {. We also let the subclass } S_{E}^{0} \text { of } S_{E} \\
& S_{E}^{0}=\left\{f=h+\bar{g} \in S_{E}: g^{\prime}(0)=B_{1}=0\right\} .
\end{aligned}
$$


The classes $S_{E}^{0}$ and $S_{E}$ were first studied in [11]. Also, we let $K_{E}^{0}, S_{E}^{*, 0}$ and $C_{E}^{0}$ denote the subclasses of $S_{E}^{0}$ of harmonic functions which are, respectively, convex, starlike and close-toconvex in U. For definitions and properties of these classes, one may refer to $([11,12])$ or [13].

For $0 \leq \alpha<1, m \in N$ and $n \in N_{0}=\{0,1, \ldots\}$ let

$$
\begin{aligned}
& N_{E}(\alpha)=\left\{f \in E: \operatorname{Re} \frac{f^{\prime}(z)}{z^{\prime}} \geq \alpha, z=r e^{i \theta} \in U\right\}, \\
& G_{E}(\alpha)=\left\{f \in E: \operatorname{Re}\left\{\left(1+\rho e^{i \gamma}\right) \frac{D^{m} f(z)}{D^{n} f(z)}-\rho e^{i \gamma}\right\} \geq \alpha, \gamma \in R, z \in U\right\},
\end{aligned}
$$

where $z^{\prime}=\frac{\partial}{\partial \theta}\left(z=r e^{i \theta}\right), f^{\prime}(z)=\frac{\partial}{\partial \theta} f\left(r e^{i \theta}\right)$

Define $T N_{E}(\alpha)=N_{E}(\alpha) \cap T$ and $T G_{E}(\alpha)=G_{E}(\alpha) \cap T$, where $T$ consists of the functions $f=h+\bar{g}$ in $S_{E}$ so that $h$ and $g$ are of the form

$$
h(z)=z-\sum_{k=2}^{\infty}\left|A_{k}\right| z^{k}, \quad g(z)=\sum_{k=1}^{\infty}\left|B_{k}\right| z^{k} .
$$

The classes $N_{E}(\alpha)$ and $G_{E}(\alpha)$ were initially introduced and studied, respectively, in [14, 15]. A function in $G_{E}(\alpha)$ is called Goodman-Salagean-type harmonic univalent function in $U$.

In this paper, we will frequently use the notations

$$
\begin{aligned}
& \Omega(f)=\Omega\left(\begin{array}{lll}
a_{1}, & b_{1}, & c_{1} \\
a_{2}, & b_{2}, & c_{2}
\end{array}\right) f, \\
& D_{k-1}=\frac{\left(\left|a_{1}\right|\right)_{k-1}\left(\left|b_{1}\right|\right)_{k-1}}{\left(\left|c_{1}\right|\right)_{k-1}(1)_{k-1}}, \quad E_{k-1}=\frac{\left(\left|a_{2}\right|\right)_{k-1}\left(\left|b_{2}\right|\right)_{k-1}}{\left(\left|c_{2}\right|\right)_{k-1}(1)_{k-1}},
\end{aligned}
$$

and a well-known formula

$$
F(a, b ; c ; 1)=\frac{\Gamma(c-a-b) \Gamma(c)}{\Gamma(c-a) \Gamma(c-b)}, \operatorname{Re}(c-a-b)>0 .
$$

In this paper the main object is to establish some important connections between the classes $K_{E}^{0}, S_{E}^{*, 0}, C_{E}^{0}, N_{E}(\alpha)$ and $G_{E}(\alpha)$ by applying the convolution operator.

\section{Connections with Goodman-Salagean-type Harmonic Univalent Functions}


In order to establish connections between harmonic convex functions, we need following results in Lemma 1 [11], Lemma 2 [15] and Lemma 4 [10].

Lemma 1. If $f=h+\bar{g} \in K_{E}^{0}$ where $h$ and $g$ are given by Eqn. (3) with $B_{1}=0$, then

$$
\left|A_{n}\right| \leq \frac{n+1}{2} \quad, \quad\left|B_{n}\right| \leq \frac{n-1}{2}
$$

Lemma 2. Let $f=h+\bar{g}$ be given by Eqn. (3). If

$$
\begin{aligned}
\sum_{k=2}^{\infty}[[(1 & \left.\left.+\rho) k^{m}-k^{n}(\alpha+\rho)\right]\left|a_{k}\right|+\left[(1+\rho) k^{m}-(-1)^{m-n} k^{n}(\alpha+\rho)\right]\left|b_{k}\right|\right] \\
& \leq 1-\alpha
\end{aligned}
$$

then $f$ is sense-preserving, Goodman-Salagean-type harmonic univalent functions in $\mathrm{U}$ and $f \in$ $G_{E}(\alpha)$.

Remark 3. In [15], it is also shown that $f=h+\bar{g}$ given by Eqn. (5) is in the family $T G_{E}(\alpha)$, if and only if the coefficient condition (6) holds. Moreover, if $f \in T G_{E}(\alpha)$, then

$$
\begin{aligned}
& \left|A_{k}\right| \leq \frac{1-\alpha}{(1+\rho) k^{m}-k^{n}(\alpha+\rho)}, \quad k \geq 2, \\
& \left|B_{k}\right| \leq \frac{1-\alpha}{(1+\rho) k^{m}-(-1)^{m-n} k^{n}(\alpha+\rho)} \quad, \quad k \geq 1 .
\end{aligned}
$$

Lemma 4. If $a, b, c>0$, then

(i) $F(a+n, b+n ; c+n ; 1)=\frac{(c)_{n}}{(c-a-b-n)_{n}} F(a, b ; c ; 1)$, for $n=0,1,2,3, \ldots$, if $c>a+b+\mathrm{n}$.

(ii) $\sum_{k=2}^{\infty}(k-1) \frac{(a)_{k-1}(b)_{k-1}}{(c)_{k-1}(1)_{k-1}}=\frac{a b}{c-a-b-1} F(a, b ; c ; 1)$, if $c>a+b+1$.

(iii) $\sum_{k=2}^{\infty}(k-1)^{2} \frac{(a)_{k-1}(b)_{k-1}}{(c)_{k-1}(1)_{k-1}}=\left[\frac{(a)_{2}(b)_{2}}{(c-a-b-2)_{2}}+\frac{a b}{c-a-b-1}\right] F(a, b ; c ; 1)$, if $c>a+b+2$.

(iv) $\sum_{k=2}^{\infty}(k-1)^{3} \frac{(a)_{k-1}(b)_{k-1}}{(c)_{k-1}(1)_{k-1}}=\left[\frac{(a)_{3}(b)_{3}}{(c-a-b-3)_{3}}+\frac{3(a)_{2}(b)_{2}}{(c-a-b-2)_{2}}+\frac{a b}{c-a-b-1}\right] F(a, b ; c ; 1)$, if $c>a+b+3$.

Theorem 5. Let $a_{i}, b_{i} \in C \backslash\{0\}, c_{i} \in R$ and $c_{i}>\left|a_{i}\right|+\left|b_{i}\right|+2$ for $i=1$, 2. If for some $\rho(0 \leq \rho \leq 1)$ and $\alpha(0 \leq \alpha<1)$, when $m=1, n=0$ the inequality 


$$
Q_{1} F\left(\left|a_{1}\right|,\left|b_{1}\right| ; c_{1} ; 1\right)+R_{1} F\left(\left|a_{2}\right|,\left|b_{2}\right| ; c_{2} ; 1\right) \leq 4(1-\alpha)
$$

is satisfied, then $\Omega\left(K_{E}^{0}\right) \subset G_{E}(\alpha)$, where

$$
\begin{aligned}
& Q_{1}=(1+\rho) \frac{\left(\left|a_{1}\right|\right)_{2}\left(\left|b_{1}\right|\right)_{2}}{\left(c_{1}-\left|a_{1}\right|-\left|b_{1}\right|-2\right)_{2}}-(3+2 \rho-\alpha) \frac{\left|a_{1} b_{1}\right|}{\left(c_{1}-\left|a_{1}\right|-\left|b_{1}\right|-1\right)}+2(1-\alpha) \\
& R_{1}=(1+\rho) \frac{\left(\left|a_{2}\right|\right)_{2}\left(\left|b_{2}\right|\right)_{2}}{\left(c_{2}-\left|a_{2}\right|-\left|b_{2}\right|-2\right)_{2}}+(1+2 \rho+\alpha) \frac{\left|a_{2} b_{2}\right|}{\left(c_{2}-\left|a_{2}\right|-\left|b_{2}\right|-1\right)} .
\end{aligned}
$$

Proof. Let $f=h+\bar{g} \in K_{E}^{0}$ where $h$ and $g$ are of the form Eqn. (3) with $B_{1}=0$. We need to show that $\Omega(f)=H+\bar{G} \in G_{E}(\alpha)$, where $H$ and $G$ defined by Eqn. (4) are analytic functions in $\mathrm{U}$. In view of Lemma 2 , we need to prove that $P_{1} \leq 1-\alpha$ where

$$
\begin{aligned}
P_{1}= & \sum_{k=2}^{\infty}\left[(1+\rho) k^{m}-k^{n}(\alpha+\rho)\right]\left|\frac{\left(a_{1}\right)_{k-1}\left(b_{1}\right)_{k-1}}{\left(c_{1}\right)_{k-1}(1)_{k-1}} A_{k}\right| \\
& +\sum_{k=2}^{\infty}\left[(1+\rho) k^{m}-(-1)^{m-n} k^{n}(\alpha+\rho)\right]\left|\frac{\left(a_{2}\right)_{k-1}\left(b_{2}\right)_{k-1}}{\left(c_{2}\right)_{k-1}(1)_{k-1}} B_{k}\right|
\end{aligned}
$$

In view of Lemma 1 and Lemma 4, it follows that

$$
\begin{aligned}
& P_{1} \leq \frac{1}{2} \sum_{k=2}^{\infty}(k+1)\left[(1+\rho) k^{m}-k^{n}(\alpha+\rho)\right] D_{k-1} \\
&+\frac{1}{2} \sum_{k=2}^{\infty}(k-1)\left[(1+\rho) k^{m}-(-1)^{m-n} k^{n}(\alpha+\rho)\right] E_{k-1} \\
&=\frac{1}{2} \sum_{k=2}^{\infty}\left[(1+\rho)(k-1)^{2}-(3+2 \rho-\alpha)(k-1)+2(1-\alpha)\right] D_{k-1} \\
& \quad+\frac{1}{2} \sum_{k=2}^{\infty}\left[(1+\rho)(k-1)^{2}+(1+2 \rho+\alpha)(k-1)\right] E_{k-1} \\
&=\frac{1}{2} Q_{1} F\left(\left|a_{1}\right|,\left|b_{1}\right| ; c_{1} ; 1\right)+\frac{1}{2} R_{1} F\left(\left|a_{2}\right|,\left|b_{2}\right| ; c_{2} ; 1\right)-(1-\alpha) .
\end{aligned}
$$

Hence $P_{1} \leq 1-\alpha$ follows from the given condition.

In order to determine connection between $T N_{E}(\beta)$ and $G_{E}(\alpha)$, we need the following results in Lemma 6 [14] and Lemma 8 [3] . 
Lemma 6. Let $f=h+\bar{g}$ where $h$ and $g$ are given by Eqn. (5) with $B_{1}=0$, and suppose that $0 \leq \beta<1$. Then

$$
f \in \operatorname{TN}_{E}(\beta) \Leftrightarrow \sum_{k=2}^{\infty} k\left|A_{k}\right|+\sum_{k=2}^{\infty} k\left|B_{k}\right| \leq 1-\beta
$$

Remark 7. If $f \in T N_{E}(\beta)$, then

$$
\left|A_{k}\right| \leq \frac{1-\beta}{k}, \quad k \geq 2, \quad\left|B_{k}\right| \leq \frac{1-\beta}{k}, \quad k \geq 1
$$

Lemma 8. Let $a, b \in C \backslash\{0\}, a \neq 1, b \neq 1, c \in(0,1) \cup(1, \infty)$ and $c>\max \{0,|a|+|b|-$ 1\}. Then

$$
\sum_{k=1}^{\infty} \frac{1}{k} \frac{(|a|)_{k-1}(|b|)_{k-1}}{(c)_{k-1}(1)_{k-1}}=\frac{(c-|a|-|b|)}{(|a|-1)(|b|-1)} F(|a|,|b| ; c ; 1)-\frac{(c-1)}{(|a|-1)(|b|-1)} .
$$

Theorem 9. Let $a_{i}, b_{i} \in C \backslash\{0\}, a_{i} \neq 1, b_{i} \neq 1, c_{i} \in R$ and $c_{i}>\max \left\{0,\left|a_{i}\right|+\left|b_{i}\right|-1\right\}$ for $i=1,2$. If for some $\beta(0 \leq \beta<1)$ and $\alpha(0 \leq \alpha<1)$, when $m=1, n=0$ and $m=2, n=0$ and $m=2, n=1$ the inequality

$$
\begin{aligned}
Q_{2} F\left(\left|a_{1}\right|,\left|b_{1}\right| ; c_{1} ; 1\right)+R_{2} F\left(\left|a_{2}\right|,\left|b_{2}\right| ; c_{2} ; 1\right) & \leq \frac{(1-\alpha)(2-\beta)}{(1-\beta)} \\
& -((\alpha+\rho))\left[\frac{\left(c_{1}-1\right)}{\left(\left|a_{1}\right|-1\right)\left(\left|b_{1}\right|-1\right)}-\frac{\left(c_{2}-1\right)}{\left(\left|a_{2}\right|-1\right)\left(\left|b_{2}\right|-1\right)}\right]
\end{aligned}
$$

is satisfied, then $\Omega\left(T N_{E}(\beta)\right) \subset G_{E}(\alpha)$, where

$$
\begin{aligned}
& Q_{2}=(1+\rho)-(\alpha+\rho) \frac{\left(c_{1}-\left|a_{1}\right|-\left|b_{1}\right|\right)}{\left(\left|a_{1}\right|-1\right)\left(\left|b_{1}\right|-1\right)^{\prime}} \\
& R_{2}=(1+\rho)+(\alpha+\rho) \frac{\left(c_{2}-\left|a_{2}\right|-\left|b_{2}\right|\right)}{\left(\left|a_{2}\right|-1\right)\left(\left|b_{2}\right|-1\right)} .
\end{aligned}
$$

Proof. Let $f=h+\bar{g} \in T N_{E}(\beta)$ where $h$ and $g$ are given by Eqn. (5). In view of Lemma 2 , it is enough to show that $P_{2} \leq 1-\alpha$ and

$$
P_{2}=\sum_{k=2}^{\infty}\left[(1+\rho) k^{m}-k^{n}(\alpha+\rho)\right]\left|\frac{\left(a_{1}\right)_{k-1}\left(b_{1}\right)_{k-1}}{\left(c_{1}\right)_{k-1}(1)_{k-1}} A_{k}\right|
$$




$$
+\sum_{k=1}^{\infty}\left[(1+\rho) k^{m}-(-1)^{m-n} k^{n}(\alpha+\rho)\right]\left|\frac{\left(a_{2}\right)_{k-1}\left(b_{2}\right)_{k-1}}{\left(c_{2}\right)_{k-1}(1)_{k-1}} B_{k}\right| .
$$

Using Remark 7 and Lemma 8 if $m=1, n=0$. Then

$$
\begin{aligned}
P_{2} & \leq(1-\beta)\left(\sum_{k=2}^{\infty}\left[(1+\rho)-\frac{(\alpha+\rho)}{k}\right] D_{k-1}+\sum_{k=1}^{\infty}\left[(1+\rho)+\frac{(\alpha+\rho)}{k}\right] E_{k-1}\right) \\
& =(1-\beta)\left(\begin{array}{c}
Q_{2} F\left(\left|a_{1}\right|,\left|b_{1}\right| ; c_{1} ; 1\right)+R_{2} F\left(\left|a_{2}\right|,\left|b_{2}\right| ; c_{2} ; 1\right) \\
-(1-\alpha)+\frac{(\alpha+\rho)\left(c_{1}-1\right)}{\left(\left|a_{1}\right|-1\right)\left(\left|b_{1}\right|-1\right)}-\frac{(\alpha+\rho)\left(c_{2}-1\right)}{\left(\left|a_{2}\right|-1\right)\left(\left|b_{2}\right|-1\right)}
\end{array}\right) \\
& \leq(1-\alpha)
\end{aligned}
$$

by the given hypothesis.

Now, if $m=2, n=0$, then

$$
\begin{aligned}
P_{2} \leq & (1-\beta)\left(\sum_{k=2}^{\infty}\left[(1+\rho) k-\frac{(\alpha+\rho)}{k}\right] D_{k-1}+\sum_{k=1}^{\infty}\left[(1+\rho) k-\frac{(\alpha+\rho)}{k}\right] E_{k-1}\right) \\
= & (1-\beta)\left(\sum_{k=2}^{\infty}\left[(1+\rho)(k-1)+(1+\rho)-\frac{(\alpha+\rho)}{k}\right] D_{k-1}\right. \\
& \left.\quad+\sum_{k=1}^{\infty}\left[(1+\rho)(k-1)+(1+\rho)-\frac{(\alpha+\rho)}{k}\right] E_{k-1}\right) \\
& =(1-\beta)\left(\begin{array}{c}
Q_{2} F\left(\left|a_{1}\right|,\left|b_{1}\right| ; c_{1} ; 1\right)+R_{2} F\left(\left|a_{2}\right|,\left|b_{2}\right| ; c_{2} ; 1\right) \\
-(1-\alpha)+\frac{(\alpha+\rho)\left(c_{1}-1\right)}{\left(\left|a_{1}\right|-1\right)\left(\left|b_{1}\right|-1\right)}-\frac{(\alpha+\rho)\left(c_{2}-1\right)}{\left(\left|a_{2}\right|-1\right)\left(\left|b_{2}\right|-1\right)}
\end{array}\right) \\
\leq & (1-\alpha)
\end{aligned}
$$

and

$$
\begin{aligned}
Q_{2}=(1+\rho) \frac{\left|a_{1} b_{1}\right|}{\left(c_{1}-\left|a_{1}\right|-\left|b_{1}\right|-1\right)}+(1+\rho) \frac{\left|a_{1} b_{1}\right|}{\left(c_{1}-\left|a_{1}\right|-\left|b_{1}\right|-1\right)} \\
+(\alpha+\rho) \frac{\left(c_{1}-\left|a_{1}\right|-\left|b_{1}\right|\right)}{\left(\left|a_{1}\right|-1\right)\left(\left|b_{1}\right|-1\right)} \\
R_{2}=(1+\rho) \frac{\left|a_{2} b_{2}\right|}{\left(c_{2}-\left|a_{2}\right|-\left|b_{2}\right|-1\right)}+(1+\rho) \frac{\left|a_{1} b_{1}\right|}{\left(c_{1}-\left|a_{1}\right|-\left|b_{1}\right|-1\right)}
\end{aligned}
$$




$$
+(\alpha+\rho) \frac{\left(c_{2}-\left|a_{2}\right|-\left|b_{2}\right|\right)}{\left(\left|a_{2}\right|-1\right)\left(\left|b_{2}\right|-1\right)}
$$

Finally, if $m=2, n=1$, then

$$
\begin{aligned}
P_{2} \leq & (1-\beta)\left(\sum_{k=2}^{\infty}[(1+\rho) k-(\alpha+\rho)] D_{k-1}+\sum_{k=1}^{\infty}[(1+\rho) k+(\alpha+\rho)] E_{k-1}\right) \\
= & (1-\beta)\left(\sum_{k=2}^{\infty}[(1+\rho)(k-1)-(1-\alpha)] D_{k-1}\right. \\
& \left.\quad+\sum_{k=1}^{\infty}[(1+\rho)(k-1)+(1+2 \rho+\alpha)] E_{k-1}\right) \\
& \quad(1-\beta)\left(Q_{2} F\left(\left|a_{1}\right|,\left|b_{1}\right| ; c_{1} ; 1\right)+R_{2} F\left(\left|a_{2}\right|,\left|b_{2}\right| ; c_{2} ; 1\right)-(1-\alpha)\right) \\
\leq & (1-\alpha)
\end{aligned}
$$

and

$$
\begin{aligned}
& Q_{2}=(1+\rho) \frac{\left|a_{1} b_{1}\right|}{\left(c_{1}-\left|a_{1}\right|-\left|b_{1}\right|-1\right)}-(1-\alpha), \\
& R_{2}=(1+\rho) \frac{\left|a_{2} b_{2}\right|}{\left(c_{2}-\left|a_{2}\right|-\left|b_{2}\right|-1\right)}+(1+2 \rho+\alpha) .
\end{aligned}
$$

We next find connections of the classes $S_{E}^{*, 0}, C_{E}^{0}$ and $T_{E}^{0}$ with $G_{E}(\alpha)$. However, we first need the following result which may be found in $[11,12]$ or $[16]$.

Lemma 10. If $f=h+\bar{g} \in C_{E}^{0}\left(S_{E}^{*, 0}, T_{E}^{0}\right)$ where $h$ and $g$ are given by Eqn. (3) with $B_{1}=$ 0 , then

$$
\left|A_{k}\right| \leq \frac{(2 k+1)(k+1)}{6} \quad, \quad\left|B_{k}\right| \leq \frac{(2 k-1)(k-1)}{6}
$$

Theorem 11. Let $a_{i}, b_{i} \in C \backslash\{0\}, c_{i} \in R$ and $c_{i}>\left|a_{i}\right|+\left|b_{i}\right|+3$ for $i=1,2$. If for some $\rho(0 \leq \rho \leq 1)$ and $\alpha(0 \leq \alpha<1)$, when $m=1, n=0$ the inequality

$$
Q_{3} F\left(\left|a_{1}\right|,\left|b_{1}\right| ; c_{1} ; 1\right)+R_{3} F\left(\left|a_{2}\right|,\left|b_{2}\right| ; c_{2} ; 1\right) \leq 12(1-\alpha),
$$

is satisfied, then $\Omega\left(C_{E}^{0}\right) \subset G_{E}(\alpha), \Omega\left(S_{E}^{*, 0}\right) \subset G_{E}(\alpha), \Omega\left(T_{E}^{0}\right) \subset G_{E}(\alpha)$, where

$$
Q_{3}=2(1+\rho) \frac{\left(\left|a_{1}\right|\right)_{3}\left(\left|b_{1}\right|\right)_{3}}{\left(c_{1}-\left|a_{1}\right|-\left|b_{1}\right|-3\right)_{3}}+(9+7 \rho-2 \alpha) \frac{\left(\left|a_{1}\right|\right)_{2}\left(\left|b_{1}\right|\right)_{2}}{\left(c_{1}-\left|a_{1}\right|-\left|b_{1}\right|-2\right)_{2}}
$$




$$
\begin{aligned}
& +(13+6 \rho-7 \alpha) \frac{\left|a_{1} b_{1}\right|}{\left(c_{1}-\left|a_{1}\right|-\left|b_{1}\right|-1\right)}+6(1-\alpha) \\
R_{3}=2(1 & +\rho) \frac{\left(\left|a_{2}\right|\right)_{3}\left(\left|b_{2}\right|\right)_{3}}{\left(c_{2}-\left|a_{2}\right|-\left|b_{2}\right|-3\right)_{3}}+(3+\rho-2 \alpha) \frac{\left(\left|a_{2}\right|\right)_{2}\left(\left|b_{2}\right|\right)_{2}}{\left(c_{2}-\left|a_{2}\right|-\left|b_{2}\right|-2\right)_{2}} \\
& +(1-\alpha) \frac{\left|a_{2} b_{2}\right|}{\left(c_{2}-\left|a_{2}\right|-\left|b_{2}\right|-1\right)} .
\end{aligned}
$$

Proof. Let $f=h+\bar{g} \in C_{E}^{0}\left(S_{E}^{*, 0}, T_{E}^{0}\right)$ where $h$ and $g$ are of the form Eqn. (3) with $B_{1}=0$. We need to prove that $\Omega(f)=H+\bar{G} \in G_{E}(\alpha)$, where $H$ and $G$ defined by Eqn. (4) are analytic functions in $\mathrm{U}$. In view of Lemma 2 , we need to show that $P_{3} \leq 1-\alpha$ where

$$
\begin{aligned}
P_{3}=\sum_{k=2}^{\infty} & {\left[(1+\rho) k^{m}-k^{n}(\alpha+\rho)\right]\left|\frac{\left(a_{1}\right)_{k-1}\left(b_{1}\right)_{k-1}}{\left(c_{1}\right)_{k-1}(1)_{k-1}} A_{k}\right| } \\
& +\sum_{k=2}^{\infty}\left[(1+\rho) k^{m}-(-1)^{m-n} k^{n}(\alpha+\rho)\right]\left|\frac{\left(a_{2}\right)_{k-1}\left(b_{2}\right)_{k-1}}{\left(c_{2}\right)_{k-1}(1)_{k-1}} B_{k}\right| .
\end{aligned}
$$

In view of Lemma 4 and Lemma 10, it follows that

$$
\begin{aligned}
& P_{3} \leq \frac{1}{6} \sum_{k=2}^{\infty}(2 k+1)(k+1)[(1+\rho) k-(\alpha+\rho)] D_{k-1} \\
& \quad+\frac{1}{6} \sum_{k=2}^{\infty}(2 k-1)(k-1)[(1+\rho) k+(\alpha+\rho)] E_{k-1} \\
& =\frac{1}{6} \sum_{k=2}^{\infty}\left[\begin{array}{c}
2(1+\rho)(k-1)^{3}+(9+7 \rho-2 \alpha)(k-1)^{2} \\
+(13+6 \rho-7 \alpha)(k-1)+6(1-\alpha)
\end{array}\right] D_{k-1} \\
& \quad+\frac{1}{6} \sum_{k=2}^{\infty}\left[2(1+\rho)(k-1)^{3}+(3+\rho-2 \alpha)(k-1)^{2}+(1-\alpha)(k-1)\right] E_{k-1} \\
& =\frac{1}{6} Q_{3} F\left(\left|a_{1}\right|,\left|b_{1}\right| ; c_{1} ; 1\right)+\frac{1}{6} R_{3} F\left(\left|a_{2}\right|,\left|b_{2}\right| ; c_{2} ; 1\right)-(1-\alpha) .
\end{aligned}
$$

Hence $P_{3} \leq 1-\alpha$ follows from the given condition.

In the next theorem, we establish connections between $T G_{E}(\alpha)$ and $G_{E}(\alpha)$.

Theorem 12. Let $a_{i}, b_{i} \in C \backslash\{0\}, c_{i} \in R$ and $c_{i}>\left|a_{i}\right|+\left|b_{i}\right|$ for $i=1,2$. If for some $\alpha(0 \leq \alpha<1)$, when $m=1, n=0$ the inequality

$$
F\left(\left|a_{1}\right|,\left|b_{1}\right| ; c_{1} ; 1\right)+F\left(\left|a_{2}\right|,\left|b_{2}\right| ; c_{2} ; 1\right) \leq 2
$$


is satisfied, then $\Omega\left(T G_{E}(\alpha)\right) \subset G_{E}(\alpha)$.

Proof. By using Lemma 2 and the definition of $P_{2}$ in Theorem 9, we need to prove that $P_{2} \leq 1-\alpha$.

By Remark 3, it follows that

$$
\begin{aligned}
P_{2}= & \sum_{k=2}^{\infty}\left[(1+\rho) k^{m}-k^{n}(\alpha+\rho)\right]\left|\frac{\left(a_{1}\right)_{k-1}\left(b_{1}\right)_{k-1}}{\left(c_{1}\right)_{k-1}(1)_{k-1}} A_{k}\right| \\
& \quad+\sum_{k=1}^{\infty}\left[(1+\rho) k^{m}-(-1)^{m-n} k^{n}(\alpha+\rho)\right]\left|\frac{\left(a_{2}\right)_{k-1}\left(b_{2}\right)_{k-1}}{\left(c_{2}\right)_{k-1}(1)_{k-1}} B_{k}\right| \\
\leq & (1-\alpha)\left(\sum_{k=2}^{\infty} D_{k-1}+\sum_{k=1}^{\infty} E_{k-1}\right) \\
= & (1-\alpha)\left(F\left(\left|a_{1}\right|,\left|b_{1}\right| ; c_{1} ; 1\right)+F\left(\left|a_{2}\right|,\left|b_{2}\right| ; c_{2} ; 1\right)-1\right) \\
\leq & (1-\alpha) .
\end{aligned}
$$

By the given condition, the proof is completed.

In the next results, we establish connections between $T G_{E}(\alpha)$ and $G_{E}(\alpha)$. By diluting the restrictions on the complex coefficients of Theorem 12.

Theorem 13. Let $a_{1} b_{1}<0, a_{1}, b_{1}>-1, c_{1}>\max \left\{0, a_{1}+b_{1}\right\}, a_{2}, b_{2} \in C \backslash\{0\} \quad$ and $c_{2}>\left|a_{2}\right|+\left|b_{2}\right|$, then a sufficient condition for $\Omega\left(T G_{E}(\alpha)\right) \subset G_{E}(\alpha)$ is that

$$
F\left(\left|a_{1}\right|,\left|b_{1}\right| ; c_{1} ; 1\right)-F\left(\left|a_{2}\right|,\left|b_{2}\right| ; c_{2} ; 1\right) \geq 0,
$$

for any $\rho(0 \leq \rho \leq 1)$ and $\alpha(0 \leq \alpha<1)$, when $m=1, n=0$.

Proof. Let $f=h+\bar{g} \in T G_{E}(\alpha)$ where $h$ and $g$ are of the form Eqn. (5). Then

$$
\Omega(f)=z-\sum_{k=2}^{\infty} \frac{\left(a_{1}\right)_{k-1}\left(b_{1}\right)_{k-1}}{\left(c_{1}\right)_{k-1}(1)_{k-1}}\left|A_{k}\right| z^{k}+\overline{\sum_{k=1}^{\infty} \frac{\left(a_{2}\right)_{k-1}\left(b_{2}\right)_{k-1}}{\left(c_{2}\right)_{k-1}(1)_{k-1}}\left|B_{k}\right| z^{k}} .
$$

This function can be rewritten as

$$
\Omega(f)=z+\frac{\left|a_{1} b_{1}\right|}{c_{1}} \sum_{k=2}^{\infty} \frac{\left(a_{1}+1\right)_{k-2}\left(b_{1}+1\right)_{k-2}}{\left(c_{1}+1\right)_{k-2}(1)_{k-1}}\left|A_{k}\right| z^{k}+\overline{\sum_{k=1}^{\infty} \frac{\left(a_{2}\right)_{k-1}\left(b_{2}\right)_{k-1}}{\left(c_{2}\right)_{k-1}(1)_{k-1}}\left|B_{k}\right| z^{k}} .
$$


In view of Lemma 2, we need to show that $P_{4} \leq 1$ where

$$
\begin{aligned}
P_{4}= & \frac{\left|a_{1} b_{1}\right|}{c_{1}} \sum_{k=2}^{\infty}\left[\frac{(1+\rho) k-(\alpha+\rho)}{1-\alpha}\right] \frac{\left(a_{1}+1\right)_{k-2}\left(b_{1}+1\right)_{k-2}}{\left(c_{1}+1\right)_{k-2}(1)_{k-1}}\left|A_{k}\right| \\
& \quad+\sum_{k=1}^{\infty}\left[\frac{(1+\rho) k+(\alpha+\rho)}{1-\alpha}\right] \frac{\left(a_{2}\right)_{k-1}\left(b_{2}\right)_{k-1}}{\left(c_{2}\right)_{k-1}(1)_{k-1}}\left|B_{k}\right| \\
\leq & \frac{\left|a_{1} b_{1}\right|}{c_{1}} \sum_{k=2}^{\infty} \frac{\left(a_{1}+1\right)_{k-2}\left(b_{1}+1\right)_{k-2}}{\left(c_{1}+1\right)_{k-2}(1)_{k-1}}\left|A_{k}\right|+\sum_{k=1}^{\infty} E_{k-1} \\
= & \frac{\left|a_{1} b_{1}\right|}{a_{1} b_{1}} \sum_{k=1}^{\infty} \frac{\left(a_{1}\right)_{k}\left(b_{1}\right)_{k}}{\left(c_{1}\right)_{k}(1)_{k-1}}\left|A_{k}\right|+\sum_{k=1}^{\infty} E_{k-1} \\
= & -F\left(\left|a_{1}\right|,\left|b_{1}\right| ; c_{1} ; 1\right)+F\left(\left|a_{2}\right|,\left|b_{2}\right| ; c_{2} ; 1\right)+1 \leq 1
\end{aligned}
$$

by the given condition.

In the next theorem, we present condition on the parameters $a_{1}, a_{2}, b_{1}, b_{2}, c_{1}, c_{2}$ and obtain a characterization for operator $\Omega$ which maps $T G_{E}(\alpha)$ onto itself.

Theorem 14. Let $a_{i}, b_{i}>0, c_{i}>a_{i}+b_{i}(i=1,2), \quad \rho(0 \leq \rho \leq 1)$ and $\alpha(0 \leq \alpha<1)$ when $m=1, n=0$ then $\Omega\left(T G_{E}(\alpha)\right) \subset T G_{E}(\rho, \alpha)$ if and only if

$$
F\left(\left|a_{1}\right|,\left|b_{1}\right| ; c_{1} ; 1\right)+F\left(\left|a_{2}\right|,\left|b_{2}\right| ; c_{2} ; 1\right) \leq 2 \text {. }
$$

Proof. Let $f=h+\bar{g} \in T G_{E}(\rho, \alpha)$ where $h$ and $g$ are of the form Eqn. (5). We need to prove that $\Omega(f)=H+\bar{G} \in T G_{E}(\rho, \alpha)$, where $H$ and $G$ defined by Eqn. (4) $P_{4} \leq 1$, where

$$
\begin{aligned}
P_{4}=\sum_{k=2}^{\infty} & {\left[\frac{(1+\rho) k-(\alpha+\rho)}{1-\alpha}\right]\left|\frac{\left(a_{1}\right)_{k-1}\left(b_{1}\right)_{k-1}}{\left(c_{1}\right)_{k-1}(1)_{k-1}} A_{k}\right| } \\
& +\sum_{k=1}^{\infty}\left[\frac{(1+\rho) k+(\alpha+\rho)}{1-\alpha}\right]\left|\frac{\left(a_{2}\right)_{k-1}\left(b_{2}\right)_{k-1}}{\left(c_{2}\right)_{k-1}(1)_{k-1}} B_{k}\right|
\end{aligned}
$$

By using Remark 3, we obtain

$$
P_{4} \leq \sum_{k=1}^{\infty} D_{k}+\sum_{k=0}^{\infty} E_{k} \leq F\left(\left|a_{1}\right|,\left|b_{1}\right| ; c_{1} ; 1\right)+R_{3} F\left(\left|a_{2}\right|,\left|b_{2}\right| ; c_{2} ; 1\right)-1 .
$$

Hence $P_{4} \leq 1$ follows from the given condition. 


\section{References}

[1] Hohlov, Y.E., Convolution operators preserving univalent functions, Ukrainian Mathematical Journal, 37, 220-226, 1985.

[2] de Branges, L., A proof of the Bieberbach conjecture, Acta Mathematica, 154, 137$152,1985$.

[3] Ahuja, O.P., Connections between various subclasses of planar harmonic mappings Involving hypergeometric functions, Applied Mathematics and Computation, 198 (1), 305-316, 2008.

[4] Carleson, B.C., Shaffer, D.B., Starlike and prestarlike hypergeometric functions, SIAM Journal on Mathematical Analysis, 15, 737-745, 1984.

[5] Owa, S., Srivastava, H.M., Univalent and starlike generalized hypergeometric functions, Canadian Journal of Mathematics, 39, 1057-1077, 1987.

[6] Miller, S., Mocanu, P.T., Univalence of Gaussian and confluent hypergeometric Functions, Proceedings of American Mathematical Society, 110(2), 333-342, 1990.

[7] Ruscheweyh, S., Singh, V., On the order of starlikeness of hypergeometric functions, Journal of Mathematical Analysis and Applications, 113, 1-11, 1986.

[8] Srivastava, H.M., Manocha, H.L., A Treatise on Generating Functions, Ellis Horwood Limited and John Wiley \& Sons, New York, Chichester, Toronto, 1984.

[9] Swaminathan, A., Certain Sufficiency conditions on Gaussian hypergeometric functions, Journal of Inequalities in Pure and Applied Mathematics, 5(4), Article 83, 1-10, 2004.

[10] Ahuja, O.P., Planar harmonic convolution operators generated by hypergeometric functions, Integral Transforms and Special Functions, 18 (3), 165-177, 2007.

[11] Clunie, J., Sheil-Small, T., Harmonic univalent functions, Annales Academie Scientiarum Fennice, Series A. I. Mathematica 9, 3-25, 1984.

[12] Ahuja, O.P., Planar harmonic univalent and related mappings, Journal of Inequalities in Pure and Applied Mathematics, 6(4) Art. 122, 1-18, 2005.

[13] Duren, P., Harmonic Mappings in the plane, Cambridge Tracts in Mathematics, Vol. 156, Cambridge University Press, Cambridge, 2004, ISBN 0-521064121-7.

[14] Ahuja, O.P., Jahangiri J.M., Noshiro-type harmonic univalent functions, Scientiae Mathematicae Japonicae, 6(2), 253-259, 2002.

[15] Aghalary, R., Goodman-Salagean-Type Harmonic Univalent Functions with Varying Arguments, International Journal of Mathematical Analysis, Vol. 1, no. 22, 1051-1057, 2007.

[16] Wang, X.T., Liang, X.Q., Zhang, Y.L., Precise coefficient estimates for close-toconvex harmonic univalent mappings, Journal of Mathematical Analysis and Applications, 263(2), 501-509, 2001. 